All authors: Center for Clinical Epidemiology and Biostatistics, Perelman School of Medicine, University of Pennsylvania, Philadelphia, PA.

Published online ahead of print at www.jco.org on May 11, 2015.

Supported by Grant No. R01-CA106851 from the National Cancer Institute.

Authors' disclosures of potential conflicts of interest are found in the article online at www.jco.org. Author contributions are found at the end of this article.

Clinical trial information: NCT00194363.

Corresponding author: Kathryn $\mathrm{H}$. Schmitz, PhD, MD, Perelman School of Medicine, University of Pennsylvania 423 Guardian Dr, 8th Floor, Blockley Hall, Philadelphia, PA 19104; e-mail: schmitz@mail.med.upenn.edu.

() 2015 by American Society of Clinical Oncology

0732-183X/15/3319w-2184w/\$20.00 DOI: 10.1200/JCO.2014.57.7395

\title{
Weight Lifting and Physical Function Among Survivors of Breast Cancer: A Post Hoc Analysis of a Randomized Controlled Trial
}

Justin C. Brown and Kathryn H. Schmitz

$$
\begin{array}{llllllll}
\text { A } & \text { B } & \text { S } & \text { T } & \text { R } & \text { A } & \text { C } & \text { T }
\end{array}
$$

\section{Purpose}

Survivors of breast cancer may experience deterioration of physical function. This is important because poor physical function may be associated with premature mortality, injurious falls, bone fracture, and disability. We conducted a post hoc analysis to explore the potential efficacy of slowly progressive weight lifting to reduce the incidence of physical function deterioration among survivors of breast cancer.

\section{Methods}

Between October 2005 and August 2008, we conducted a single-blind, 12-month, randomized controlled trial of twice-per-week slowly progressive weight lifting or standard care among 295 survivors of nonmetastatic breast cancer. In this post hoc analysis of data from the Physical Activity and Lymphedema Trial, we examined incident deterioration of physical function after 12 months, defined as a $\geq 10$-point decrease in the physical function subscale of the Medical Outcomes Short-Form 36-item questionnaire.

\section{Results}

The proportion of participants who experienced incident physical function deterioration after 12 months was $16.3 \%(24 / 147)$ in the control group and $8.1 \%(12 / 148)$ in the weight lifting group (relative risk, $0.49 ; 95 \% \mathrm{Cl}, 0.25$ to $0.96 ; P=.04$ ). No serious or unexpected adverse events occurred that were related to weight lifting.

\section{Conclusion}

Slowly progressive weight lifting compared with standard care reduced the incidence of physical function deterioration among survivors of breast cancer. These data are hypothesis generating. Future studies should directly compare the efficacy of weight lifting with other modalities of exercise, such as brisk walking, to appropriately inform the development of a confirmatory study designed to preserve physical function among survivors of breast cancer.

\section{J Clin Oncol 33:2184-2189. (c) 2015 by American Society of Clinical Oncology}

\section{INTRODUCTION}

There are more than 3.1 million survivors of breast cancer in the United States. ${ }^{1}$ The median age at diagnosis of breast cancer is 61 years. ${ }^{1}$ Earlier detection and improved curative therapy have resulted in $79 \%$ and $73 \%$ of survivors of early-stage breast cancer living 10 and 20 years after diagnosis, respectively. ${ }^{2}$ Favorable long-term survival rates translate to a growing population of survivors of breast cancer who will live into old age and subsequently experience adverse age-related health outcomes, such as the deterioration of physical function. ${ }^{3}$

Physical function is the ability to complete activities required for safe independent living. ${ }^{4}$ In a recent prospective cohort study, there were no base- line differences in functional status when comparing 6,390 women who never had a cancer diagnosis with the 374 women who had experienced a diagnosis of breast cancer sometime during the 10-year follow-up period. Women diagnosed with breast cancer reported an accelerated trajectory of deterioration of physical function after diagnosis compared with members of the cancer-free cohort of similar age. $^{5}$ This observation is supported by reports that the physiologic systems necessary to sustain physical function, ${ }^{6}$ such as the cardiopulmonary and musculoskeletal systems, ${ }^{7-9}$ are impaired among survivors of breast cancer when compared with age-matched controls. ${ }^{10,11}$ Frailty is a syndrome characterized by the simultaneous decline in multiple physiologic systems that yields a high susceptibility to adverse 
health outcomes, such as disability. ${ }^{12}$ Survivors of breast cancer may be at increased risk for developing frailty at a younger age compared with cancer-free controls. ${ }^{13}$ These observations are important because poor physical function may be associated with premature mortality among survivors of breast cancer. ${ }^{14-17}$ In addition, poor physical function may be associated with injurious falls, bone fracture, and disability among survivors of breast cancer. ${ }^{18-20}$ Collectively, these observational data suggest that survivors of breast cancer may experience deterioration of physical function and that poor physical function may be associated with adverse health outcomes.

It is unknown whether an intervention to increase muscular strength through the use of slowly progressive weight lifting is an efficacious modality to reduce the incidence of deterioration of physical function among survivors of breast cancer. To test the hypothesis that weight lifting would reduce the incidence of deterioration of physical function, we conducted a post hoc analysis using data from the Physical Activity and Lymphedema (PAL) trial, a randomized controlled trial designed to determine the safety of weight lifting among survivors of breast cancer.

\section{METHODS}

\section{Participants}

The primary aim of the PAL trial was to assess the safety of slowly progressive weight lifting among survivors of breast cancer with breast cancerrelated lymphedema $(\mathrm{n}=141)$ and survivors of breast cancer at risk for breast cancer-related lymphedema $(\mathrm{n}=154)$. The primary outcomes of the PAL trial were published separately for survivors of breast cancer with lymphedema ${ }^{21}$ and survivors of breast cancer at risk for lymphedema. ${ }^{22}$ The analysis described herein includes survivors of breast cancer with lymphedema and survivors of breast cancer at risk for lymphedema $(n=295)$. A detailed description of the PAL trial methods are described elsewhere. ${ }^{23}$ Survivors of breast cancer were recruited through the metropolitan Philadelphia region. ${ }^{24}$ Participants were eligible for the study if they were: 1) female survivors 1 to 15 years after diagnosis; 2) free from cancer at study entry; 3 ) $\geq 1$ lymph node(s) removed; and 4) no medical conditions or contraindicated medications that would prohibit participation in an exercise program. Additional eligibility criteria included 5) body mass index $\left.(\mathrm{BMI}) \leq 50 \mathrm{~kg} / \mathrm{m}^{2} ; 6\right)$ no plans for surgery during the study; 7) no history of bilateral lymph node removal; 8) no weight lifting in the previous year; and 9) stable body weight and not attempting to lose weight.

Study participants were randomly assigned to one of two study groups through the use of minimization. ${ }^{23}$ Participants were randomly assigned to one of the two study groups after being stratified according to the following baseline variables: lymphedema status (diagnosed or not diagnosed), age ( $<54$ years or $\geq 54$ years), difference in lymphedema arm volume $(<10 \%$ or $10 \%$ to $20 \%$ or $>20 \%$ ), number of lymph nodes removed $(<6$ or $\geq 6$ ), obesity (BMI $<30$ or $\geq 30 \mathrm{~kg} / \mathrm{m}^{2}$ ), and time since diagnosis ( $<60$ months or $\geq 60$ months).

This trial was approved by the University of Pennsylvania institutional review board. All participants provided written informed consent and written clearance from a physician before participating in any study-related activities.

\section{Intervention}

Study participants randomly assigned to the weight lifting group were provided with a 12-month membership to a community fitness center, most commonly YMCA, proximal to their residence. For the first 13 weeks, participants were given instruction on the safe completion of weight lifting exercises in small groups of between two and six participants. Certified exercise professionals employed by the fitness centers led the twice-per-week exercise sessions that each lasted 90 minutes. Each session included stretching of major muscle groups, low-intensity aerobic warmup, abdominal and back strengthening exercises, and weight lifting exercises. Weight lifting exercises for the upper body included the dumbbell press, seated row, lateral or front raise, bicep curl, and triceps extension. Weight lifting exercises for the lower body included the leg press, back extension, leg extension, and leg curl. One to three new weight lifting exercises were taught at each session. For each exercise session, three sets of each weight lifting exercise were performed using 10 repetitions per set. For each exercise, weight was progressively increased after two sessions at which three sets of 10 repetitions could be performed at a given weight without concurrent changes in arm and hand symptoms. No maximal upper limit was placed on the weight lifted for each exercise. After 13 weeks of supervised weight lifting, participants were instructed to continue participating in unsupervised weight lifting for 39 weeks, adhering to the same exercise prescription used during the supervised portion of the trial. Weight lifting adherence was quantified using attendance logs completed by study participants and verified for completion by the exercise professionals. Adherence was defined for this report as attendance to weight lifting sessions. The exercise professionals contacted study participants if they missed more than one exercise session each week throughout the year. Participants in the control group were asked to not change their physical activity volume during the study. Upon study completion, control group participants were offered a 12-month membership to a community fitness center with 13 weeks of supervised exercise instruction similar to that of the weight lifting group.

\section{Safety}

All community-based certified exercise professionals underwent a 3-day training course that reviewed the exercise protocol and outlined lymphedema management practices. Study participants in both randomly assigned groups with diagnosed lymphedema received custom-fitted compression garments (Jobst, BSN Medical, Hamburg, Germany) at baseline and at 6 months. Participants in the weight lifting group with diagnosed lymphedema were required to wear their study-provided lymphedema garments during all weight lifting activities. The certified exercise professionals systematically asked all participants about any changes in symptoms each week and measured arm circumference and volume each month to identify any changes in arm swelling. All study participants were required to complete a 1-hour educational lecture about lymphedema risk reduction, management, and exercise safety that was based on the National Lymphedema Network clinical practice guidelines (http://www.lymphnet.org/resources).

\section{Measurements}

Measurements were obtained from all participants at baseline and at 12 months by trained staff who followed a standardized protocol and were blinded to study group assignments. Before completing the measurement visit, all study participants were reminded not to disclose their study group to the measurement staff.

Demographic characteristics-including age, education, race, and smoking habits-were self-reported at baseline. Clinical characteristics including time since cancer diagnosis and cancer stage were collected from the state cancer registry, surgical pathology report, or self-report. The presence and severity of lymphedema was quantified using water displacement volumetry. ${ }^{23}$ Cancer treatment therapies including chemotherapy, radiation, and endocrine therapies were self-reported.

Muscular strength of the upper and lower body was quantified using one-repetition maximum (1-RM) testing with bench press and leg press exercises. 1-RM testing is the maximum amount of weight that can be lifted one time. 1-RM tests are the gold standard for evaluating muscular strength. ${ }^{25,26}$ 1-RM tests are safe for clinical populations with appropriate conduct and supervision. ${ }^{27,28}$ Participants completed a warmup set of four to six repetitions using a weight of $2.25 \mathrm{~kg}$ on the bench press and $18.2 \mathrm{~kg}$ on the leg press and then rated exertion using a scale from 1 to 10 . Participant rating established the first weight at which a 1-RM test was attempted. Additional weight was added until exercise biomechanics were compromised or the participant was unwilling or unable to try a heavier weight. Anthropometric measures included height, weight, and whole-body dual-energy x-ray absorptiometry to quantify fat mass and lean mass (Hologic Discovery, Bedford, MA). Caloric intake was quantified using the Diet History Questionnaire. ${ }^{29}$ Physical activity was assessed using the International Physical Activity Questionnaire. ${ }^{30}$ 
Table 1. Baseline Characteristics of Study Participants, According to Study Group

\begin{tabular}{|c|c|c|c|}
\hline Characteristic & $\begin{array}{l}\text { Weight } \\
\text { Lifting } \\
\text { (n=148) }\end{array}$ & $\begin{array}{l}\text { Control } \\
(n=147)\end{array}$ & $P$ \\
\hline Age, years (mean $\pm S D$ ) & $55.3 \pm 8.5$ & $56.7 \pm 9.1$ & .28 \\
\hline Education, No. (\%) & & & .46 \\
\hline High school or less & $20(14)$ & $27(18)$ & \\
\hline Some college & $54(36)$ & $47(32)$ & \\
\hline College degree or more & $74(50)$ & $73(50)$ & \\
\hline Race, No. (\%) & & & .06 \\
\hline White & $90(61)$ & $101(69)$ & \\
\hline Black & $47(32)$ & $43(29)$ & \\
\hline Other & $11(7)$ & $3(2)$ & \\
\hline Smoking habits, No. (\%) & & & .40 \\
\hline Never & $74(50)$ & $85(58)$ & \\
\hline Former & $66(45)$ & $55(37)$ & \\
\hline Current & $8(5)$ & 7 (5) & \\
\hline $\begin{array}{l}\text { Time since cancer diagnosis, months } \\
\text { (mean } \pm \text { SD) }\end{array}$ & $57.9 \pm 38.1$ & $63.7 \pm 40.3$ & .11 \\
\hline Cancer stage, No. (\%) & & & .34 \\
\hline Ductal carcinoma in situ & $1(1)$ & $0(0)$ & \\
\hline I & $69(47)$ & $63(43)$ & \\
\hline$\|$ & $3(2)$ & $0(0)$ & \\
\hline III & $44(30)$ & $48(33)$ & \\
\hline Unknown & $31(21)$ & $36(24)$ & \\
\hline Lymphedema, No. (\%) & $71(48)$ & $70(48)$ & .99 \\
\hline \multicolumn{4}{|l|}{ Cancer treatments, No. (\%) } \\
\hline Chemotherapy & 115 (78) & $109(74)$ & .50 \\
\hline Radiation & $118(80)$ & $111(76)$ & .41 \\
\hline Tamoxifen & $29(20)$ & 19 (13) & .30 \\
\hline Aromatase inhibitor & $1(1)$ & $1(1)$ & .99 \\
\hline $\begin{array}{l}\text { Physical functioning (scale, } 0-100 \\
\text { [mean } \pm \text { SD]) }\end{array}$ & $79.8 \pm 19.6$ & $80.4 \pm 19.6$ & .76 \\
\hline
\end{tabular}

\section{Outcome}

The outcome of this post hoc analysis was a deterioration of $\geq 10$ points on the physical function subscale of the Medical Outcomes Short-Form 36item (SF-36) questionnaire after 12 months. ${ }^{31}$ A deterioration of $\geq 10$ points in physical function has been noted as being clinically meaningful ${ }^{32,33}$ and is associated with an increase in the risk of premature mortality among survivors of breast cancer. ${ }^{16}$ The physical function subscale of the SF-36 questionnaire asks about the ability to complete 10 tasks including moderate- and vigorousintensity activities, lifting groceries, climbing stairs, and walking various distances ranging from one block to more than one mile. The composite physical function subscale is scored by averaging the 10 responses, with each response ranging from 0 to 100 and higher values indicating better physical function.

\section{Statistical Analysis}

All statistical analyses were completed using Stata MP Version 13.1 (StataCorp, College Station, TX). Descriptive statistics presented for baseline variables include counts and proportions for categorical variables and means and standard deviations for continuous variables. Categorical variables were compared between the two study groups using Fisher's exact test, and continuous variables were compared between the two study groups using the Wilcoxon rank-sum test. Data on participants who were missing physical function measures at 12 months were imputed through the use of a multiple imputation procedure that included baseline physical function as well as demographic, clinical, and anthropometric variables. ${ }^{34}$ We calculated the relative risk (RR) and 95\% CI using an unadjusted generalized linear model. The outcome of a $\geq 10$-point decrease on the physical function subscale of the SF-36 questionnaire was not prespecified in the PAL trial protocol. We conceptualized this

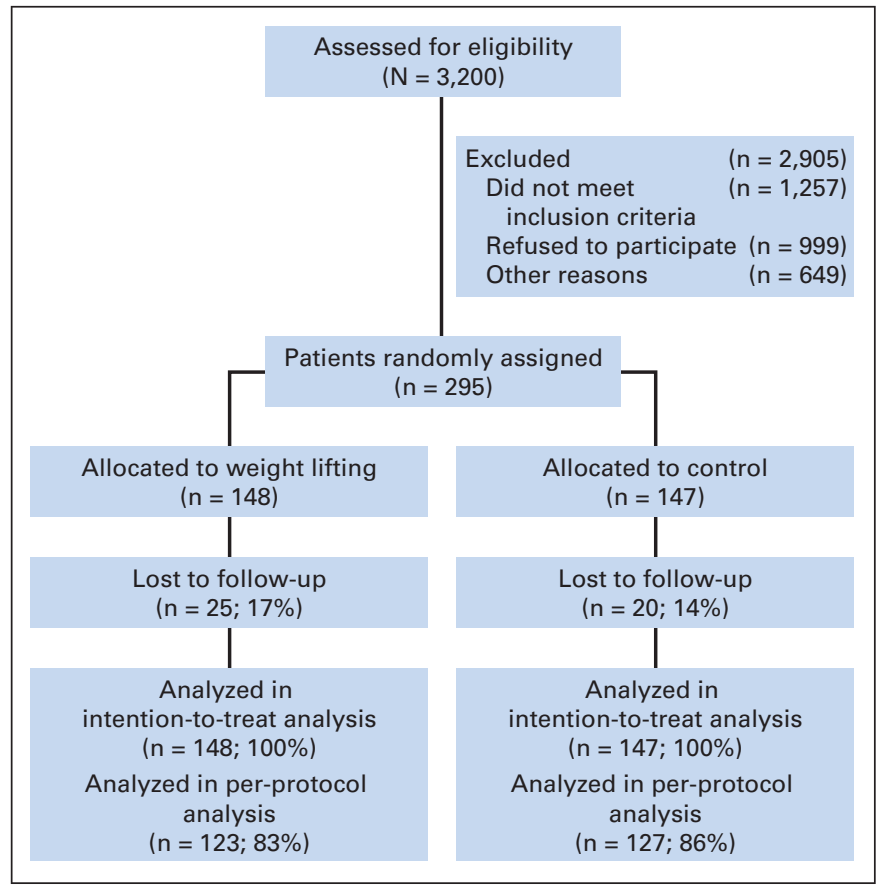

Fig 1. CONSORT diagram of enrollment, random assignment, and follow-up of study participants.

outcome and our analysis after it was reported that a decrease of $\geq 10$ points in physical function may be associated with an increase in the risk of premature mortality among survivors of breast cancer. ${ }^{16}$ Therefore, our results should be interpreted cautiously and as hypothesis generating.

\section{RESULTS}

Between October 2005 and February 2007, 295 survivors of breast cancer were recruited and randomly assigned with data collection ending for all study participants in August 2008. Characteristics of the study participants at baseline are presented in Table 1 . Physical function scores at baseline were $79.8 \pm$ a standard deviation of 19.6 and $80.4 \pm 19.6$ in the weight lifting and control groups, respectively $(P=$ .76). The mean baseline physical function score of the 295 trial participants was not significantly different from previously published values of physical function among survivors of breast cancer $(P=.25){ }^{35}$

The rates of attrition over 12 months were similar between the two study groups (Fig $1 ; P=.52$ ). Participants who did not complete the 12-month assessment were more likely to be younger $(51.2 \pm 8.8 \mathrm{v}$ $56.8 \pm 8.6$ years; $P<.001)$, have greater upper body strength $(20.9 \pm$ $7.9 v 18.3 \pm 5.7 \mathrm{~kg} ; P=.04)$, have greater lower body strength $(88.7 \pm$ $26.5 v 77.0 \pm 24.8 \mathrm{~kg} ; P=.003)$, and have a higher BMI $(31.1 \pm 6.1 v$ $\left.28.5 \pm 6.1 \mathrm{~kg} / \mathrm{m}^{2} ; P=.008\right)$ at baseline. Baseline physical function was not significantly different between participants who did not complete the 12-month assessment compared with those who did complete the 12-month assessment (scores, $78.8 \pm 18.8 v 80.3 \pm 18.8 ; P=.71$ ).

Strength, anthropometric, diet, and physical activity characteristics are presented in Table 2. At baseline, 1-RM bench press strength ranged from 0.0 to $43.1 \mathrm{~kg}$, and 1-RM leg press strength ranged from 11.3 to $186.0 \mathrm{~kg}$. The median adherence rates to the weight lifting protocol were $96 \%, 88 \%, 73 \%$, and $65 \%$ in the first, second, third, and 


\begin{tabular}{|c|c|c|c|c|c|c|}
\hline \multirow[b]{2}{*}{ Variable } & \multicolumn{3}{|c|}{ Baseline } & \multicolumn{3}{|c|}{12 Months } \\
\hline & $\begin{array}{l}\text { Weight Lifting } \\
(\text { mean } \pm \text { SD) }\end{array}$ & $\begin{array}{c}\text { Control } \\
(\text { mean } \pm \text { SD })\end{array}$ & $P$ & $\begin{array}{l}\text { Weight Lifting } \\
(\text { mean } \pm \text { SD) }\end{array}$ & $\begin{array}{c}\text { Control } \\
(\text { mean } \pm \text { SD })\end{array}$ & $P$ \\
\hline Bench press, maximum, $\mathrm{kg}$ & $19.1 \pm 6.6$ & $18.4 \pm 5.7$ & .40 & $24.1 \pm 6.9$ & $18.5 \pm 5.4$ & $<.001$ \\
\hline Leg press, maximum, kg & $79.8 \pm 25.6$ & $77.8 \pm 25.3$ & .61 & $100.8 \pm 26.7$ & $80.9 \pm 25.6$ & $<.001$ \\
\hline Weight, kg & $79.1 \pm 16.5$ & $79.3 \pm 17.4$ & .85 & $77.3 \pm 15.8$ & $78.4 \pm 17.1$ & .78 \\
\hline $\mathrm{BMI}, \mathrm{kg} / \mathrm{m}^{2}$ & $29.0 \pm 5.9$ & $28.7 \pm 6.4$ & .55 & $28.4 \pm 5.5$ & $28.6 \pm 6.4$ & .97 \\
\hline Body fat, \% & $37.8 \pm 5.7$ & $38.4 \pm 5.8$ & .36 & $37.4 \pm 5.9$ & $38.7 \pm 5.8$ & .08 \\
\hline Fat mass, $\mathrm{kg}$ & $30.6 \pm 10.0$ & $31.1 \pm 1.6$ & .82 & $29.6 \pm 9.6$ & $31.1 \pm 1.5$ & .29 \\
\hline Lean mass, kg & $48.5 \pm 7.5$ & $48.1 \pm 7.6$ & .54 & $47.7 \pm 7.5$ & $47.3 \pm 7.6$ & .60 \\
\hline Diet, kcal/day & $1,731.1 \pm 1,227.9$ & $1,653.3 \pm 1,304.8$ & .77 & $1,511.7 \pm 74.0$ & $1,443.0 \pm 75.2$ & .35 \\
\hline Physical activity, MET-hours/week & $64.0 \pm 66.9$ & $59.2 \pm 63.6$ & .19 & $65.2 \pm 56.7$ & $58.8 \pm 74.5$ & .05 \\
\hline
\end{tabular}

fourth quarters of the study, respectively. After 12-months, participants in the weight lifting group increased upper and lower body strength compared with the control group, as reflected by 1-RM bench press strength $(5.0 \pm 0.8 v 0.1 \pm 0.7 \mathrm{~kg} ; P<.001)$ and $1-\mathrm{RM}$ leg press strength $(21.0 \pm 3.2 v 3.1 \pm 3.1 \mathrm{~kg} ; P<.001)$, respectively. Participants in the weight lifting group reported more physical activity compared with the control group after 12 months $(P=.05)$. No other differences in anthropometric or diet characteristics were noted between the two study groups after 12 months.

The proportion of participants who experienced incident deterioration of physical function after 12 months was $16.3 \%(24 / 147)$ in the control group and $8.1 \%(12 / 148)$ in the weight lifting group (RR, 0.49; 95\% CI, 0.25 to $0.96 ; P=.04$; Table 3 ). These results are based on imputed data following an intention-to-treat analysis; these findings did not differ when the analysis was repeated without imputation following a perprotocol analysis (RR, $0.49 ; 95 \% \mathrm{CI}, 0.25$ to $0.97 ; P=.04$ ).

There were no serious adverse events related to the weight lifting intervention. Nonserious adverse events, including musculoskeletal injuries that required dose modification or cessation of weight lifting have been reported elsewhere in detail. ${ }^{36}$

\section{DISCUSSION}

The principal finding from this secondary analysis is that survivors of breast cancer who participated in slowly progressive weight lifting for
12 months were less likely to experience incident deterioration of physical function compared with control group participants $(8.1 \%$ [12/148] v 16.3\% [24/147]; $P=.04)$.

For each 10-point decrease in physical function among survivors of breast cancer, the risk of premature mortality has been estimated to increase by $6 \%{ }^{16}$ The association between deterioration of physical function and premature mortality has also been reported among Medicare beneficiaries. ${ }^{37}$ A deterioration of $\geq 10$ points in physical function is similar to the quality-of-life burden experienced as a result of having congestive heart failure or chronic lung disease. ${ }^{33}$ Therefore, the preservation of physical function among survivors of breast cancer may hold clinical importance. The findings from our trial provide preliminary evidence to support the potential efficacy of weight lifting to promote the health and longevity of breast cancer survivorship by preserving physical function.

There have been several other randomized trials of weight lifting among survivors of breast cancer. ${ }^{38-40}$ These studies have consistently demonstrated that moderate-intensity slowly progressive weight lifting during and after chemotherapy is feasible and safe. These studies have examined a variety of end points including lymphedema arm volume, body composition, quality of life, and mean changes in physical function. However, to our knowledge, no such study has demonstrated that weight lifting prevents the deterioration of physical function compared with standard care using a $\geq 10$-point decrease on the physical function subscale of the SF-36 questionnaire. The PAL

\begin{tabular}{|c|c|c|c|c|}
\hline \multirow[b]{2}{*}{ Deterioration } & \multicolumn{2}{|c|}{ Intention-to-Treat Analysis } & \multicolumn{2}{|c|}{ Per-Protocol Analysis } \\
\hline & Weight Lifting $(n=148)$ & Control $(n=147)$ & Weight Lifting $(n=123)$ & Control $(n=127)$ \\
\hline \multicolumn{5}{|c|}{ Participants with $\geq 10$-point deterioration } \\
\hline \multicolumn{5}{|c|}{ Yes } \\
\hline Count & 12 & 24 & 11 & 23 \\
\hline$\%$ & 8.1 & 16.3 & 9.0 & 18.1 \\
\hline \multicolumn{5}{|l|}{ No } \\
\hline Count & 136 & 123 & 112 & 104 \\
\hline$\%$ & 91.9 & 83.7 & 91.0 & 81.9 \\
\hline Relative risk & \multicolumn{2}{|c|}{0.49} & \multicolumn{2}{|c|}{0.49} \\
\hline $95 \% \mathrm{Cl}$ & \multicolumn{2}{|c|}{0.25 to 0.96} & \multicolumn{2}{|c|}{0.25 to 0.97} \\
\hline$P$ & \multicolumn{2}{|c|}{.04} & \multicolumn{2}{|c|}{.04} \\
\hline
\end{tabular}


trial has been safely and efficaciously translated into the physical therapy setting. ${ }^{41}$ At our institution, it was our experience that third-party payers reimbursed the intervention using diagnosis codes for breast cancer $\left(174 . .^{*}\right)$, lymphatic disorders $\left(457 .^{\star}\right)$, joint pain and stiffness (719.*), and muscle weakness (728.9). Additional research is necessary to examine whether aerobic exercise, such as walking, is efficacious to preserve physical function among survivors of breast cancer. For example, among 1,600 older adults at risk for disability, a physical activity program focused on walking yielded an $18 \%$ reduction in the likelihood of developing major mobility disability, defined as the inability to walk 400 meters without assistance, over a follow-up period of 2.6-years. ${ }^{42}$ Additional research is also necessary to identify survivors of breast cancer who may be at risk for experiencing deterioration of physical function and may benefit from an intervention such as weight lifting or other forms of physical activity or exercise.

There are several strengths to this trial. The outcome of this study was quantified in such a way that maximizes clinical utility, representing a meaningful deterioration of physical function that is readily noticeable by patients and is associated with adverse health outcomes, such as premature mortality among survivors of breast cancer. In addition, outcome data collection was completed by staff members who were blinded to study group assignments. The sample size of the trial was diverse with respect to racial background (35\% nonwhite participants). The intervention was 12 months long with similar rates of follow-up between the two study groups. The weight lifting program successfully blended supervised and unsupervised exercise to promote safety and enable long-term sustainability.

There are several weaknesses to this trial. Physical function was not a primary outcome of the PAL trial. Consequently, participants were not recruited onto this trial on the basis of being at risk of experiencing incident deterioration of physical function. Despite our low enrollment rate, participants in the PAL trial were of similar age to women in the state cancer registry from which they were recruited. ${ }^{24}$ However, it is plausible that women who did not participate in the trial possess levels of physical function or have other characteristics that are different from those who did participate in the trial. It is noteworthy that one for every six (16.3\%) control group participants experienced incident deterioration of physical function within 12 months. We had insufficient statistical power to examine whether the benefits of slowly progressive weight lifting varied across subgroups of age, BMI, and cancer stage-all of which have been identified as factors that may influence the relationship between physical function and adverse outcomes such as mortality among survivors of breast cancer. ${ }^{14}$ This analysis was not prespecified in the PAL trial protocol and therefore should be interpreted as exploratory and hypothesis generating. In addition, we did not adjust our type-I error rate; therefore, we cannot rule out the possibility of false discovery. Despite significant increases in muscular strength, which indicates a physiologic response to weight lifting, we did not observe significant differences in lean muscle mass between the two groups at 12 months $(P=.60)$. This finding is likely a result of the dose of weight lifting that was prescribed, which sought to increase muscular strength, rather than increase muscular hypertrophy.

In conclusion, the findings from this study demonstrate the feasibility and potential efficacy for slowly progressive weight lifting to reduce incident deterioration of physical function among survivors of breast cancer. These data are hypothesis generating. Future studies should directly compare the efficacy of weight lifting with that of other modalities of exercise, such as brisk walking, for the purpose of preventing the deterioration of physical function among survivors of breast cancer. These data will be useful to inform the development of a confirmatory study to provide conclusive evidence to shape clinical practice and maximize the health and longevity among survivors of breast cancer.

\section{AUTHORS' DISCLOSURES OF POTENTIAL CONFLICTS OF INTEREST}

Disclosures provided by the authors are available with this article at www.jco.org.

\section{AUTHOR CONTRIBUTIONS}

Conception and design: All authors

Financial support: Kathryn H. Schmitz

Collection and assembly of data: Kathryn H. Schmitz

Data analysis and interpretation: All authors

Manuscript writing: All authors

Final approval of manuscript: All authors

\section{REFERENCES}

1. DeSantis $C E$, Lin $C C$, Mariotto $A B$, et al: Cancer treatment and survivorship statistics, 2014. CA Cancer J Clin 64:252-271, 2014

2. Taylor R, Davis P, Boyages J: Long-term survival of women with breast cancer in New South Wales. Eur J Cancer 39:215-222, 2003

3. Katz S, Branch LG, Branson $\mathrm{MH}$, et al: Active life expectancy. N Engl J Med 309:1218-1224, 1983

4. Jette AM: Toward a common language for function, disability, and health. Phys Ther 86:726734, 2006

5. Petrick JL, Reeve BB, Kucharska-Newton AM, et al: Functional status declines among cancer survivors: Trajectory and contributing factors. J Geriatr Oncol 5:359-367, 2014

6. Ferrucci L, Bandinelli S, Benvenuti E, et al: Subsystems contributing to the decline in ability to walk: Bridging the gap between epidemiology and geriatric practice in the InCHIANTI study. J Am Geriatr Soc 48:1618-1625, 2000

7. Morey MC, Pieper CF, Cornoni-Huntley J: Is there a threshold between peak oxygen uptake and self-reported physical functioning in older adults? Med Sci Sports Exerc 30:1223-1229, 1998

8. Shephard RJ: Maximal oxygen intake and independence in old age. $\mathrm{Br} J$ Sports Med 43:342346, 2009

9. Ferrucci L, Penninx BW, Volpato $S$, et al: Change in muscle strength explains accelerated decline of physical function in older women with high interleukin-6 serum levels. J Am Geriatr Soc 50:1947-1954, 2002

10. Jones LW, Courneya KS, Mackey JR, et al: Cardiopulmonary function and age-related decline across the breast cancer survivorship continuum. J Clin Oncol 30:2530-2537, 2012

11. Gomes PR, Freitas Junior IF, da Silva CB, et al: Short-term changes in handgrip strength, body composition, and lymphedema induced by breast cancer surgery. Revista Brasileira de Ginecologia e Obstetrícia 36:244-250, 2014

12. Fried LP, Tangen CM, Walston J, et al: Frailty in older adults: Evidence for a phenotype. J Gerontol A Biol Sci Med Sci 56:M146-M156, 2001

13. Bennett JA, Winters-Stone KM, Dobek J, et al: Frailty in older breast cancer survivors: Age, prevalence, and associated factors. Oncol Nurs Forum 40:E126-134, 2013

14. Braithwaite D, Satariano WA, Sternfeld B, et al: Long-term prognostic role of functional limitations among women with breast cancer. J Natl Cancer Inst 102:1468-1477, 2010

15. Saquib N, Pierce JP, Saquib J, et al: Poor physical health predicts time to additional breast cancer events and mortality in breast cancer survivors. Psychooncology 20:252-259, 2011

16. Sehl M, Lu X, Silliman $R$, et al: Decline in physical functioning in first 2 years after breast cancer diagnosis predicts 10-year survival in older women. J Cancer Surviv 7:20-31, 2013 
17. Marinac $C$, Patterson RE, Villasenor $A$, et al: Mechanisms of association between physical func tioning and breast cancer mortality: Evidence from the Women's Healthy Eating and Living Study. J Cancer Surviv 8:402-409, 2014

18. Hewitt M, Rowland JH, Yancik R: Cancer survivors in the United States: Age, health, and disability. J Gerontol A Biol Sci Med Sci 58:82-91, 2003

19. Winters-Stone KM, Torgrimson B, Horak F, et al: Identifying factors associated with falls in postmenopausal breast cancer survivors: A multidisciplinary approach. Arch Phys Med Rehabil 92: 646-652, 2011

20. Chen $Z$, Maricic M, Bassford TL, et al: Fracture risk among breast cancer survivors: Results from the Women's Health Initiative Observational Study. Arch Intern Med 165:552-558, 2005

21. Schmitz KH, Ahmed RL, Troxel A, et al: Weight lifting in women with breast-cancer-related lymphedema. N Engl J Med 361:664-673, 2009

22. Schmitz KH, Ahmed RL, Troxel AB, et al: Weight lifting for women at risk for breast cancerrelated lymphedema: A randomized trial. JAMA 304 2699-2705, 2010

23. Schmitz KH, Troxel AB, Cheville $A$, et al: Physical Activity and Lymphedema (the PAL trial): Assessing the safety of progressive strength training in breast cancer survivors. Contemp Clin Trials 30:233-245, 2009

24. Rogerino A, Grant LL, Wilcox H III, et al: Geographic recruitment of breast cancer survivors into community-based exercise interventions. Med Sci Sports Exerc 41:1413-1420, 2009

25. Baechle TR, Earle RW (eds): Essentials of Strength Training and Conditioning (ed 3). Champaign, IL, Human Kinetics, 2008
26. Thompson WR, Gordon NF, Pescatello LS (eds): ACSM's Guidelines for Exercise Testing and Prescription (ed 8). Philadelphia, PA, Lippincott, Williams \& Wilkins, 2010

27. Barnard KL, Adams KJ, Swank AM, et al: Injuries and muscle soreness during the one repetition maximum assessment in a cardiac rehabilitation population. J Cardiopulm Rehabil 19:52-58, 1999

28. Shaw CE, McCully KK, Posner JD: Injuries during the one repetition maximum assessment in the elderly. J Cardiopulm Rehabil 15:283-287, 1995

29. Subar AF, Thompson FE, Kipnis $V$, et al: Comparative validation of the Block, Willett, and National Cancer Institute food frequency questionnaires: The Eating at America's Table Study. Am J Epidemiol 154:1089-1099, 2001

30. Craig $C L$, Marshall $A L$, Sjöström $M$, et al: International physical activity questionnaire: 12country reliability and validity. Med Sci Sports Exerc 35:1381-1395, 2003

31. Ware JE Jr, Sherbourne CD: The MOS 36item short-form health survey (SF-36): I. Conceptual framework and item selection. Med Care 30:473483, 1992

32. Bjorner JB, Wallenstein GV, Martin MC, et al: Interpreting score differences in the SF-36 vitality scale: Using clinical conditions and functional outcomes to define the minimally important difference. Curr Med Res Opin 23:731-739, 2007

33. Alonso J, Ferrer M, Gandek B, et al: Healthrelated quality of life associated with chronic conditions in eight countries: Results from the International Quality of Life Assessment (IQOLA) Project. Qual Life Res 13:283-298, 2004

34. Rubin DB: Multiple Imputation for Nonresponse in Surveys. Hoboken, NJ, John Wiley \& Sons, 2009
35. Ganz PA, Desmond KA, Leedham B, et al: Quality of life in long-term, disease-free survivors of breast cancer: A follow-up study. J Natl Cancer Inst 94:39-49, 2002

36. Brown JC, Troxel AB, Schmitz KH: Safety of weightlifting among women with or at risk for breast cancer-related lymphedema: Musculoskeletal injuries and health care use in a weightlifting rehabilitation trial. Oncologist 17:1120-1128, 2012

37. Ware JE Jr, Bayliss MS, Rogers WH, et al: Differences in 4-year health outcomes for elderly and poor, chronically ill patients treated in $\mathrm{HMO}$ and fee-for-service systems: Results from the Medical Outcomes Study. JAMA 276:1039-1047, 1996

38. Ahmed RL, Thomas W, Yee D, et al: Randomized controlled trial of weight training and lymphedema in breast cancer survivors. J Clin Oncol 24:2765-2772, 2006

39. Courneya KS, Segal RJ, Mackey JR, et al: Effects of aerobic and resistance exercise in breast cancer patients receiving adjuvant chemotherapy: A multicenter randomized controlled trial. J Clin Oncol 25:4396-4404, 2007

40. Courneya KS, McKenzie DC, Mackey JR, et al: Effects of exercise dose and type during breast cancer chemotherapy: Multicenter randomized trial. J Natl Cancer Inst 105:1821-1832, 2013

41. Beidas RS, Paciotti B, Barg F, et al: A hybrid effectiveness-implementation trial of an evidencebased exercise intervention for breast cancer survivors. J Natl Cancer Inst Monogr 50:338-345, 2014

42. Pahor M, Guralnik JM, Ambrosius WT, et al: Effect of structured physical activity on prevention of major mobility disability in older adults: The LIFE study randomized clinical trial. JAMA 311:23872396, 2014 


\section{AUTHORS' DISCLOSURES OF POTENTIAL CONFLICTS OF INTEREST}

Weight Lifting and Physical Function Among Survivors of Breast Cancer: A Post Hoc Analysis of a Randomized Controlled Trial

The following represents disclosure information provided by authors of this manuscript. All relationships are considered compensated. Relationships are self-held unless noted. I = Immediate Family Member, Inst = My Institution. Relationships may not relate to the subject matter of this manuscript. For more information about ASCO's conflict of interest policy, please refer to www.asco.org/rwc or jco.ascopubs.org/site/ifc.

\section{Justin C. Brown}

No relationship to disclose

\section{Kathryn H. Schmitz}

No relationship to disclose 